(Aus dem physiologischen Laboratorium der Universität Leyden.)

\title{
Die Registrirung der menschlichen Herztöne mittels des Saitengalvanometers.
}

\author{
(Unter Mitwirkung von A. Flohil und P. J. T. A. Battaerd, \\ Assistenten am Laboratorium.) \\ Von
}

W. Einthoven.

(Mit 1 Textfigur und Tafel XVIII und XIX.)

Schon früher ${ }^{1}$ ) wurde im physiologischen Institut zu Leyden eine Methode, Herztöne zu registriren, ausgearbeitet, wobei als registrirendes Instrument der Capillar-Elektrometer angewendet wurde. Die damals publicirten Curven geben nicht nur den Augenblick an, wo ein Herzton anfängt oder endet ${ }^{2}$ ), sondern sie zeigen auch die von den Tönen dargebotenen Unterschiede der Qualität. Trotzdem geben sie ohne eine nähere Analyse eine durchaus ungenaue graphische Darstellung des untersuchten Schalles.

Um das richtige Bild zu erhalten, müsste man die Curven zeitraubenden Messungen unterwerfen und sich eine Anzahl von Berechnungen gefallen lassen, die wir damals nicht ausgeführt haben. Wir haben uns im Jahre 1894 mit den photographirten Bildern selbst vorläufig begnügt, weil diese schon sehr typische Formen des ersten und des zweiten Tones zeigen, so wie dieselben beim Kaninchen und an verschiedenen Stellen der Herzgegend beim Menschen zum Vorschein gebracht werden konnten.

1) W. Einthoven und M. A. J. Geluk, Die Registrirung der Herztöne. Pflüger's Arch. Bd. 57 S. 617. 1894.

2) Die Methode von Hürthle (Deutsche mediz. Wochenschr. $1893 \mathrm{Nr}$. 4) bezweckte besonders den genauen Anfang eines Herztones scharf anzugeben. Dies trifft auch für die Methode von Holowinski zu. Arch. de physiol. norm. et pathol. 5. Sér. T. 8 p. 895.1896. 
Es braucht jedoch nicht hervorgehoben zu werden, dass eine strenge graphische Darstellung den direct registrirten capillar-elektrometrischen Curven vorzuziehen ist ${ }^{1}$ ), und man kann auf einfache Weise ein schon genügendes Maass der Genauigkeit erreichen, indem man den Capillar-Elektrometer dureh das viel empfindlichere und zugleich schneller reagirende Saitengalvanometer ersetzt.

Inwiefern das Saitengalvanometer im Stande ist, schnelle Schwingungen richtig wiederzugeben, ist schon an anderer Stelle ${ }^{2}$ ) näher auseinandergesetzt worden. Aus unserer Sammlung von versilberten Quarzfäden ist Nr. 20 der leichteste. Dieser ist $2,5 \mathrm{~cm}$ lang und $1 \mu$ dick, während er ungefähr $1,5 \times 10^{-7} \mathrm{~g}$ wiegt, ein so geringes Gewicht, dass es keiner Wage einen merklichen Ausschlag zu geben vermag. Der Faden kann wie eine Violinsaite gespannt werden und zeigt dann Schwingungen einer Periode von $0,31 \sigma$. Diese Periode entspricht derjenigen eines Tones von 3230 ganzen Schwingungen pro Sekunde, - also ungefähr gis ${ }^{4}$ oder fast dem höchsten Ton des gewöhnlichen Pianos.

Ohne die Gefahr des Reissens kann die Spannung der Saite noch erhöht werden. Auch kann ein kürzerer Faden genommen werden, so dass man nach Belieben über noch grössere Schwingungsfrequenzen verfügen kann.

Es wäre aber gänzlich unnöthig, dieselben behufs der Registrirung der Herztöne anzuwenden. Wir brauchen kaum daran zu erinnern, dass die Tonhöhe der durch die Herzwirkung erzeugten Schälle viel niedriger ist als die oben erwähnte, und dass unsere gedämpft schwingende Saite von so hohem Eigentone die ihr mitgetheilte periodische Bewegung der niedrigen Herztöne so gut wie fehlerlos wiedergeben muss.

Wenn man jedoch schon behaupten darf, dass der Quarzfaden im Saitengalvanometer im Stande ist, den Luftschmingungen des Herztones genau zu folgen, so ist damit noch nicht gesagt, dass alle

1) Vor Kurzem hat Otto Frank dies noch nachdrücklich betont, siehe Münchener mediz. Wochenschr. 51. Jahrg. Nr. 22. 1904. Frank hat die Herztöne erfolgreich direct mechanisch registrirt; seine Curven sind aber, insofern mir bekannt ist, noch nicht publicirt worden. - Ueber einen Versuch, die Herztöne mechanisch zu registriren, vgl. auch $\mathrm{Hürthle,} \mathrm{Zentralbl.} \mathrm{f.} \mathrm{Physiol.} \mathrm{Bd.} 18$ S. 617. 1904 .

2) Vgl. verschiedene Aufsätze in Drude's Annalen der Physik $1903 \mathrm{ff}$. und in "Onderzoekingen" physiol. Laborat. Leyden II. Reihe. 
Die Registrirung der menschl. Herztöne mittels des Saitengalvanometers. 463

beim Registriren vorkommenden Schwierigkeiten gelöst sind. Bei der von uns angewandten Methode müssen die Luftschwingungen des zu registrirenden Schalles erst die Grundplatte eines Mikrophons der mittels eines constanten Stromgebers bleibend durchströmt wird - in Bewegung versetzen. Die Schwingungen der Grundplatte erzeugen Intensitätsschwankungen des Mikrophonstroms, und es bilden diese Stromschwankungen, nachdem sie mittels eines Transformators zum Saitengalvanometer geleitet worden sind, die Ursache der Hin- und Herbewegungen des Quarzfadens. Ausser auf die Eigenschaften des letzteren muss man also noch auf diejenigen des Mikrophons und des Transformators Rücksicbt nehmen.

Die Mikrophone, wie sie in Handel vorkommen, entsprechen, was ihre Fähigkeit betrifft, sehr frequente Schwingungen wiederzugeben, zwar nicht ebenso hohen Ansprüchen wie das Saitengalvanometer, aber für unseren Zweck, die Registrirung der Herztöne, genügt schon ein bei vielen Telephongesellschaften üblicher Apparat von Berliner. Auch die Construction eines zweckentsprechenden Transformators ruft keine Schwierigkeiten hervor. Wir haben einen eisenfreien regulirbaren Transformator auf Hartgummischeiben absichtlich für diesen Zweck anfertigen lassen, konnten jedoch mit relativ nicht viel Verlust auch einen gewöhnlichen Inductionsapparat von du Bois-Reymond oder Helmholtz anwenden, wenn nur das Eisen aus der primären Rolle entfernt worden war. Die von uns ausgeführte Berechnung, den für den Zweck günstigsten Transformator zu erhalten, mag hier wegbleiben und besser gelegentlich bei der Besprechung der Registrirung sehr hoher Töne ausführlich mitgetheilt werden.

Die untenstehende Figur veranschanlicht, wie der Schall eines Herztones zum Mikrophon hingeleitet wird.

Der Mikrophon $M$ ist in der Mitte eines ziemlich schweren, in der Figur nicht gezeichneten eisernen Ringes befestigt, der nach der Methode von Julius an drei Punkten erschütterungsfrei aufgehängt ist. Die drei Stahldrähte, an denen der Ring hängt, sind an ihrem unteren Ende mit stählernen Federn versehen, wodurch die Erschütterung sowohl in horizontaler wie in verticaler Richtung aufgehoben wird.

$B$ ist eine umgebogene Metallröhre mit einem Querstücke $Z$, worin sich der Habn $K$ befindet. Die Röhre $B$ selbst ist fest mit 
einem isolirten Pfeiler oder einer Wandtafel verbunden und mit ihrem einen Ende durch den Kautschukschlauch $c_{1}$ an dem Mikrophon, mit ibrem anderen Ende durch den Kautschukschlauch $c_{2}$ an dem Stethoskoptrichter $T$ befestigt. Letztgenannter Kautschukschlauch ist ungefähr $75 \mathrm{~cm}$ lang. Man kann den Stethoskoptrichter in die Hand nehmen und hin und her bewegen, ohne dass das Mikrophon die geringste Spur einer Erschütterung zeigt.

Man beurtheilt dies mittels eines Quecksilberspiegels, dessen Runzeln ein sehr empfindliches Reagens für Erschütterungen ist, und womit man die Zweckmässigkeit der Julius'schen Methode in jedem Augenblick während des Versuches leicht beweist.

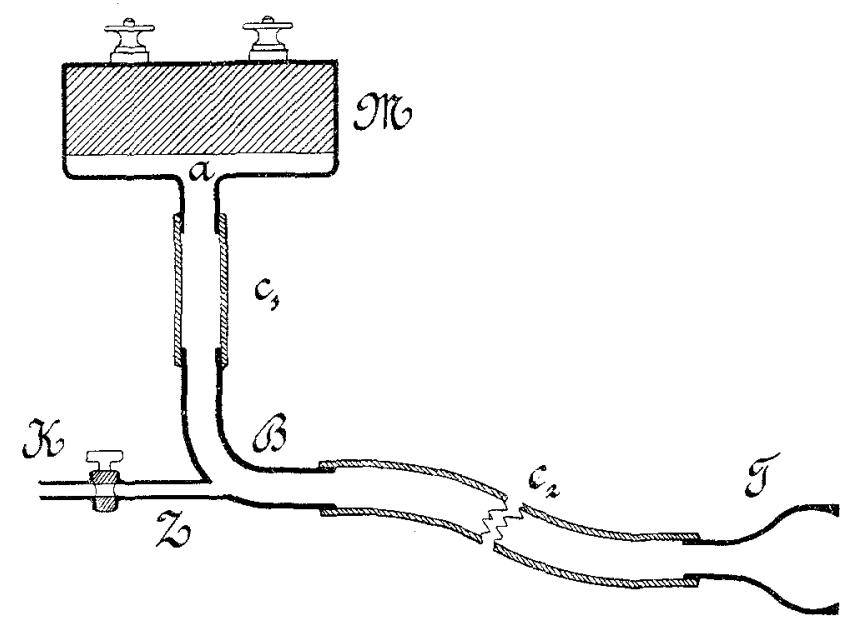

Der Hahn $Z$ wird immer maximal geöffnet gehalten, so dass der Luftdruck im Röhrensystem $c_{\mathbf{1}} B c_{\mathbf{2}}$ dem atmosphärischen Luftdruck gleich bleibt. Unter diesen Umständen ist ein Ueberbringen der Stösse und Erschütterungen vom Stethoskop zum Mikrophon gänzlich ausgeschlossen und kann letzterer nur durch einen Schall in der Form von Luftschwingungen getroffen werden.

Auf den Taf. XVIII und XIX sind eine Anzahl saitengalvanometrischer Photogramme von Herztönen wiedergegeben ${ }^{1}$ ). Der angewendete Quarzfaden Nr. 15 war nicht so leicht wie Nr. 20, er wurde aber immer stark gespannt und entsprach den gestellten Anforderungen

1) Wir sind gerne bereit, den Fachgenossen auf Anfrage Copien unserer Negative zu übersenden. 
Die Registrirung der menschl. Herztöne mittels des Saitengalvanometers. 465

in genügendem Maasse. Während des Registrirens wurde ebenso wie bei all unseren früher mittels des Saitengalvanometers erhaltenen Curven nach der Methode Garten's ${ }^{1}$ ) ein Netz von Quadratmillimetern auf der empfindlichen Platte entworfen, wodurch eine später auszuführende Messung der photographirten Herztöne bedeutend erleichtert wird.

Die Umdrehungsgeschwindigkeit unseres Speichenrades ist sehr constant und stimmt stets genau mit dem im voraus bestimmten Betrag überein. Die Abweichungen sind kleiner als 1:1000, so dass - ausser an einigen regelmässig wiederkehrenden Stellen, die den zufälliger Weise verschobenen Speichen entsprechen - die zwischen den Ordinaten vorhandenen Distanzen vollkommen zuverlässige Zeitmaasse darstellen.

Die Bewegungsgeschwindigkeit der empfindlichen Platte war jedoch während der Versuchsreihe meistens zu gross, wodurch die Ordinatdistanzen grösser als $1 \mathrm{~mm}$ geworden sind. Obgleich dieser Umstand keinen Einfluss auf die Genauigkeit der Zeitmessung ausübt, sind die Apparate jetzt behufs später folgender Versuche doch insofern verändert, dass auch diese Geschwindigkeit der empfindlichen Platte besser regulirt werden kann.

Bei den bier reproducirten Photogrammen haben wir zwei verschiedene Geschwindigkeiten angewandt: In den Fig. 1 und 2 der Taf. XVIII und 8 und 9 der Taf. XIX entspricht ein Skalentheil auf der Abscisse 0,04 Secunden, während in den übrigen Figuren ein Skalentheil auf der Abscisse einen Werth von 0,02 Secunden hat.

Man kann der empfindlichen Platte auch grössere Geschwindigkeiten, z. B. bis auf $1000 \mathrm{~mm}$ per Secunde ${ }^{2}$ ), mittheilen. Aber wenn man grosse Geschwindigkeiten anwendet, um die Form der Schallwellen deutlicher abzubilden, so geht die Möglichkeit, eine Anzahl von Herztönen innerhalb eines kleinen Raumes registriren zu können, verloren.

Die Unterschiede zwischen den in diesem Aufsatz abgebildeten Curven und denjenigen, die früher mittels des Capillarelektrometers

1) Dr. Si g fried Garten. Abhandl. d. kgl. sächs. Gesellsch. d. Wissenschaft. zu Leipzig, math.-phys. Classe Bd. 26 Nr. 5 S. 381. 1901.

2) Siehe z. B. die Figuren 12 und 13 in Archives internation. de Physiol. vol. 4 p. 141 und 142. 1906. 
erhalten wurden, fallen in die Augen. Wir brauchen hierbei nicht länger zu verweilen und begnügen uns damit, darauf hinzuweisen, dass unsere Curven sich durch die nahezu symmetrische Form unterhalb und oberhalb der Nulllinie unterscheiden.

Die Fig. 1, 2, 3 und 4 stellen die Spitzentöne vier verschiedener Personen dar. Man sieht leicht, welche Saitenschwingungen dem systolischen und welche dem diastolischen Ton entsprechen. Zum Ueberfluss sind diese noch in der Figur mit 1 und 2 markirt, während mittels einer Klammer, die vom Anfang des ersten bis zum Anfang des zweiten Tones reicht, die Daner der Systole angedeutet wird.

Betrachtet man die genannten Photogramme näher, so fallen erstens einige Eigenschaften auf, die sie mit einander gemein haben. Man sieht, dass der erste Ton bei allen untersuchten Personen kräftiger ist und eine längere Dauer hat als der zweite. Eine Tonhöhe kann nicht bestimmt werden, weil die untersuchten Schälle unregelmässig sind und viel besser mit kurzdauernden Geräuschen als mit musikalischen Klängen verglichen werden können. Aber wie unregelmässig der Schall auch sein mag, für jede Person ist er charakteristisch. Vergleicht man z. B. die ersten Töne der verschiedenen Herzperioden der Fig. 1 mit einander, so bemerkt man so viele gegenseitige Uebereinstimmung, dass es den Schein hat, als. seien sie alle nur Copien eines einzigen Modelles. Das hat auch für die diastolisehen Töne Geltung, und obgleich deutliche Unterschiede zwischen den Figuren der verschiedenen Personen vorhanden sind, so trifft doch für jede einzelne Figur dieselbe Bemerkung zu wie für Fig. 1.

Bei der Beurtheilung der Unterschiede, welche die vier Figuren unter einander darbieten, nuuss man auf zwei Umstände Rücksicht nehmen. Erstens war, wie oben schon bemerkt wurde, die Geschwindigkeit der empfindlichen Platte während des Photographirens von Fig. 3 und 4 zweimal grösser als bei den Figuren 1 und 2. Und weiter sind die zum Galvanometer hingeleiteten elektrischen Ströme bei der Anfertigung der vier Photogramme in verschiedenem Grade abgeschwächt worden. Dies hat zur Folge, dass man aus dem Unterschiede der Amplitudines der Saitenschwingungen in den vier erwähnten Photogrammen nicht auf einen Intensitätsuntersehied der Herztöne schliessen darf. Die saitengalvanometrische Vorrichtung war dermaassen empfindlich, dass, wenn man die Herztöne in voller 
Stärke zu registriren wünschte, das Saitenbild aus dem Gesichtsfeld herausgeschlagen werden würde. Bei allen Photogrammen mussten also die Amplitudines der Saitenbewegung mehr oder. weniger verkleinert werden. Namentlich bei jungen, mageren Personen mit dünner Brustwand, deren Herztöne laut hörbar sind, wie z. $B$ bei Mo Fig. 3, und weiter auch oft in pathologischen Fällen, ist man genöthigt, die Saitenbewegungen bedeutend zu reduciren.

Die Reduction kann auf verschiedene Weise stattfinden, und es wäre nicht schwierig, hierbei feste Maasse anzuwenden, so dass aus den Dimensionen der erhaltenen Curven und dem Betrage der angewandten Reduction immer die wirklichen Intensitäten hergeleitet werden könnten. Vorläufig haben wir diese jedoch nicht berechnet und uns mit einer Abschwächung des Mikrophonstroms bis auf einen solchen Betrag begnügt, dass zweckentsprechende Amplitudines der Saitenbewegung erzielt wurden.

Die Intensitätsverhältnisse der verschiedenen Schälle in ein em selben Photogramme können dahingegen unmittelbar aus der Frequenz und der Amplitude der Saitenschwingungen hergeleitet werden, und zwar ist die Stärke jedes Tones proportional dem Producte aus dem Quadrat der Schwingungsfrequenz und dem Quadrat der Amplitude.

Beim weiteren Studium der Unterschiede, die zwischen den Spitzentönen der vier untersuchten Personen vorhanden sind, achte man ausser auf die Schwingungsfrequenz und die allgemeine Form der Schälle noch auf das Verhältniss der Schwingungsamplitude in ersten und zweiten Ton, auf die Dauer der Töne und auf die Dauer von Systole und Diastole.

In den Figuren 5 (Taf. XVIII) und 6 (Taf. XIX) sind die Pulmonaltöne von zwei Personen wiedergegeben, so wie man dieselben hart am Sternum im zweiten Intercostalraum bört. Die Unterschiede von den Spitzentönen kommen deutlich zum Vorschein. Der erste Ton hat eine kürzere Dauer sowobl in absolutem Maasse wie auch im Verhältniss zur Dauer des zweiten Tones. Namentlich von diesem letzteren Ergebniss überzeugt man sich schon mit einem Blick.

In Fig. 5 ist die Phase zwischen dem ersten und dem zweiten Ton nicht ganz ohne Schwingung, während doch die auscultatorische Untersuchung bei Herrn $B a$, der ein gesunder junger Mann mit normaler Herzwirkung ist, kein systolisches Geräusch am Ostium pulmonale aufweist. Diese. Schwingungen zwischen erstem und 
zweitem Ton sind in der Figur um so mehr auffallend, weil der Quarzfaden in der Herzpause jedesmal so gut wie vollkommen zur Ruhe kommt.

In Fig. 6 des Herrn $O u$ ist im Gegensatz zu Fig. 5 der zweite Ton deutlich stärker als der erste, während in der Phase zwischen erstem und zweitem Ton eben so wenig Schwingungen zu erkennen sind wie in der Herzpause.

Wir schliessen unseren Aufsatz mit dem Hinweis auf die Figuren 7, 8, 9 und 10 der Taf. XIX. Sie stellen die Herztöne und Herzgeräusche dreier Patienten des Universitäts-Krankenhauses zu Leyden dar. ${ }^{1}$ ) Während die Patienten sich im Krankenbause befanden, wurden ihre Herztöne im physiologischen Laboratorium, d. h. auf ungefähr $1,5 \mathrm{~km}$ Distanz, mittels des Saitengalvanometers registrirt. Auf welche Weise diese beiden Gebäude mittels elektriseher Leitungsdrähte mit einander verbunden sind, inwiefern die Genauigkeit der zu erzielenden graphisehen Bilder durch den Gebrauch des Verbindungskabels herabgesetzt wird, und bis auf wie viel Kilometer die Distanz zwischen dem Patienten und dem Registrirapparat vergrössert werden darf, bevor die zu erzielenden Ergebnisse dadurch bedeutend beeinträchtigt werden, ist vor Kurzem in einem Aufsatze über das Telecardiagramm näher entwickelt worden. ${ }^{2}$ ) Wir kommen darauf jetzt nicht zurück und gehen besser unmittelbar zur Besprechung der Photogramme über.

Fig. 7 bildet die Pulmonaltöne eines Patienten ab, der ein deutlich hörbares systolisehes Geräusch hat. Im Photogramm geht der erste Ton allmählich in ein Geräusch über; es ist kein deutlicher Unterschied zwischen dem ersten Ton und dem Geräusch bemerkbar, während der zweite Ton, der kräftiger als der erste ist, sich scharf von dem Geräusche abhebt, so dass man den Zeitpunkt des Anfanges der Diastole mit ziemlich grosser Genauigkeit angeben kann. Weiter darf noch bemerkt werden, dass in der Herzpause nahezu keine Saitenschwingungen sichtbar sind.

In Fig. 8 sieht man die Schälle abgebildet, die an der Herzspitze einer Patientin mit Stenose und Insufficienz der Mitralklappen

1) Es ist uns recht angenehm, an dieser Stelle Herrn Professor Nolen und seinen Assistenten für die uns immer bereitwillig geleistete Hülfe unseren herzlichen Dank abzustatten.

2) Siehe Arch. internat. de Physiol. vol. 4 p. 132. 1906. Auch in „Onderzoekingen" physiol. Laborat. Leyden II. Reihe. 
wahrgenommen werden können. Bei dieser Patientin fühlt man an der Herzspitze ein präsystolisches Schwirren, während das Stethoskop uns das dortige Vorhandensein eines präsystolischen und eines systolischen Geräusches offenbart. Auf dem Photogramm, wo beide Geräusche deutlich ans Licht kommen, ist das erste mit $a$ näher angerlentet.

In Fig. 9 findet man die Pulmonaltöne derselben Patientin wiedergegeben. Man sieht, dass das präsystolische und das systolische Geräusch hier nicht oder kaum mehr vorbanden sind, während es weiter noch in die Augen fällt, dass der zweite Ton im Verhältniss zum ersten nur schwach ist. Die Frage, ob der zweite Pulmonalton verstärkt sei oder nicht, - die eine klinische Bedeutung hat muss oftmals bei alleiniger anscultatorischer Untersuchung ungelöst hleiben, und auch in dem speciellen Falle unserer Patientin war die Antwort zweifelhaft geblieben. Wegen der wechselnden anatomischen Verhältnisse des Thorax wird man sich keine Mühe geben, die absolute Intensität des Schalles zu erforschen, sich aber mit einer Vergleichung der Tonstärken, z. B. zwischen denjenigen des zweiten Pulmonaltones und des ersten Tones desselben Ostiums oder zwischen denjenigen des zweiten Pulmonaltones und des zweiten Aortatones begnügen. Aber auch diese Vergleichungen sind für das wahrnehmende Ohr mit nicht geringer Schwierigkeit verbunden, da man namentlich mit Schällen von verschiedener Dauer und versehiedenem Typus zu thun hat ${ }^{1}$ ).

Unter diesen Umständen darf von der Registrirung der Herztöne eine wesentliche Hülfe erwartet werden. Betrachtet man Fig. 9 unserer Patientin neben den Fig. 5 und 6 , welche die Pulmonaltöne von ein paar gesunden jungen Männern mit normalen Herzen wiedergeben, so ist man ohne einige Zurückhaltung zu der Behauptung berechtigt, dass der zweite Pulmonalton bei der Patientin wenigstens im Verhältniss zu dem ersten Tone desselben Ostiums nicht verstärkt ist.

1) Nach Max Wien, Pflüger's Arch. Bd. 97 S. 1. 1903, sollen niedrige Töne von 50 Schwingungen pro Secunde objektiv viele Millionen Male stärker sein als hohe Töne von 2000 Schwingungen pro Secunde, um eine gleich starke Schallempindung zu erzielen. H. Zwa ardemaker und F. H. Quix geben in Engelmann's Arch. f. Physiol. S. 25. 1904, Unterschiede an, deren Form principiell mit den obengenannten übereinstimmt, aber deren Betrag von einer anderen Ordnung ist. 
Während die Curven der bisher besprochenen Figuren leicht in Uebereinstinınung mit den auscultatorischen, mittels des Stethoskops verrichteten Beobachtungen gebracht werden konnten, bietet die Erklärung der jetzt am Schluss noch zu erwähnenden Fig. 10 uns grosse Schwierigkeiten dar. In dieser Figur wird der Schall, der an der Herzspitze eines jungen Mannes mit Mitralinsufficienz und starker Hypertrophie der beiden Herzhälften hörbar ist, wiedergegeben. Mittels des Stethoskops nimmt man an der Spitze ein stark gedehntes, ziemlich gleichförmiges systolisches Geräusch wahr, dessen Anfang nicht scharf angegeben werden kann, das jedoch plötzlich nit dem zwar einigermaassen dumpfen, aber doch nicht schwachen diastolischen Ton endigt. Zwisehen dem diastolisehen Ton und dem Anfang des darauf folgenden systolischen Geräusches ist eine deutliche Pause vorbanden.

Diese Beschreibung stimmt nicht zur Form der in Fig. 10 abgebildeten Curve. Die Figur zeigt für jede Herzperiode ausser der Pause fünf deutlich von einander zu unterscheidende Schälle, die wir mit $a, s_{1}, s_{2}, s_{3}$ und 2 angedentet haben. Welche Bedeutung jeder dieser Schälle hat, und in welcher Reihenfolge sie in der Periode vorkommen, können wir nicht mit Gewissheit entscheiden. Unsere Erfahrung ist dafür noch zu gering; wir zweifeln aber nicbt daran, dass man in der Folge durch weitere Untersuchungen die "Cardiophotogramme" - wie unsere Curven heissen mögen - auf genügende Weise wird erklären können. Für die Deutung der jetzigen Fig. 10 wagen wir vorläufig die folgende Hypothese:

$$
\begin{array}{ll}
a & =\text { präsystolisches Geräusch, } \\
s_{1}, s_{2}, s_{3} & =\text { drei Phasen des systolischen Geräusches, } \\
2 & =\text { diastolischer Ton, } \\
p & =\text { Pause, }
\end{array}
$$

während dann die in der Figur angebrachte Klammer die ganze Periode begrenzen mag.

Eine der Schwierigkeiten, die Figur zu deuten, liegt in der Dauer des schallfreien Stadiums jeder Herzperiode. Dieses Stadium scheint bei der Untersuchung mittels des Stethoskopes ziemlich lange zu dauern, während es im Photogramm nur äusserst kurz zum Vorschein kommt. Wünscht man die mangelhafte Uebereinstimmung. zwischen den beiden Beobachtungsmethoden den Fehlern des Registrirens zuzuschreiben, so müsste man annehmen, dass ein zufälliger Schall die schreibende Saite gerade im schallfreien Stadium der 
Herzwirkung in Schwingung versetzt hätte. Aber diese Annabme muss als sehr unwahrscheinlich verworfen werden, weil ja die mehr als sieben nach einander folgenden Herzperioden des Photogrammes an Gleichförmigkeit nur wenig zu wünsehen übrig lassen.

Unseres Erachtens tragen die Măngel des Ohres die Schuld. Vielleicht wird nur darum so regelmässig eine Pause gebört, weil das Ohr unmittelbar vorher durch den ziemlich starken Schall des diastolischen Tones getroffen wird, wodurch es temporär ermüdet wird. Die Stille, die man hier wahrnimmt, wäre dann mit der scheinbaren Stille vergleichbar, die stets unmittelbar der Erzeugung eines starken Knalles in einer übrigens schwach rauschenden Umgebung zu folgen scheint.

Es wurde oben schon darauf hingewiesen, dass das Ohr ein unzuverlässlicher Führer bei der Beurtheilung der Schallintensität ist, und es ist unnöthig, an noch mehr Mängel dieses Organes zu erinnern, um uns in der Ueberzeugung zu bestärken, dass der Forscher mit Vortheil die Registrirung der Herztöne benutzen kann. Wir meinen damit keineswegs, dass die gewöbnliche Auscultation am Krankenbette durch die Registrirmethode ersetzt werden solle, sondern nur, dass letztere mit gutem Erfolg zur Ergänzung der ersteren angewandt werden kann.

Die Frage, ob das Registriren der Herztöne je eine klinische Methode werden wird oder vielleicht auch jetzt sehon so genannt werden darf, ist selbstverständlich von den Anforderungen abhängig, die man an eine klinische Methode stellen zu müssen glaubt. Hier treffen dieselben Betrachtungen $\mathrm{zu}$, die wir schon am Schluss unseres Aufsatzes über das Telecardiogramm ${ }^{1}$ ) erörterten. Das Saitengalvanometer ist, verglichen mit vielen einfachen klinischen Apparaten, ein ziemlich komplicirtes und kostspieliges Instrument, das eine besondere Aufstellung erfordert und nur mit Hülfe geübter Assistenz gebraucht werden kann, so dass es vom Kliniker selbst, während er beim Patienten sitzt, vorläufig wohl nicht zur Hand genommen werden wird. Aber dem gegenüber steht, dass vom Beobachter nur Kenntniss des von ihm gebrauchten Instrumentariums und gar keine specielle Geschicklichkeit gefordert wird, während so gut wie jede photographische Aufnahme gelingt und innerhalb weniger Minuten abgelaufen ist.

1) A. a. 0 . 
472 W. Einthoven: Die Registrirung der menschl. Herztöne etc.

Mögen die für das Registriren benöthigten Instrumente auch nicht im Krankensaal am Platze sein, durch eine zweckentsprechende Verbindung mittels elektrischer Leitungsdrähte wird diese Schwierigkeit gelöst. Während die Verbindung zwischen dem physiologisehen Institut und dem Universitäts-Krankenhaus zu Leyden jetzt seit ungefähr 2 Jabren gebraucht wird, hat sie noch niemals eine Störung erfahren, und wo der Physiologe und der Kliniker zu gemeinschaftlicher Arbeit vereinigt werden können, steht der klinischen Anwendung der Herztöne-Registrirung weiter auch nichts im Wege. 

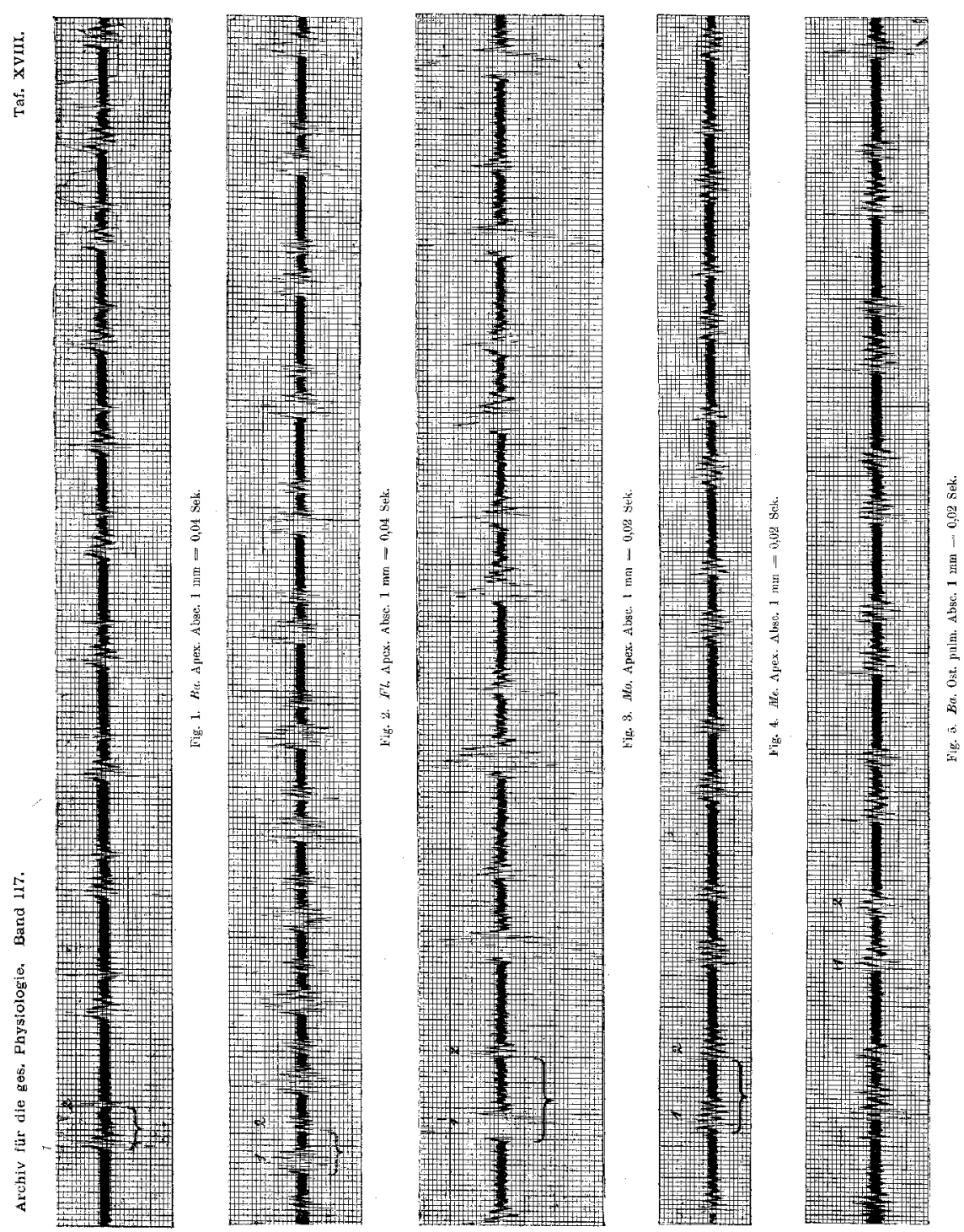

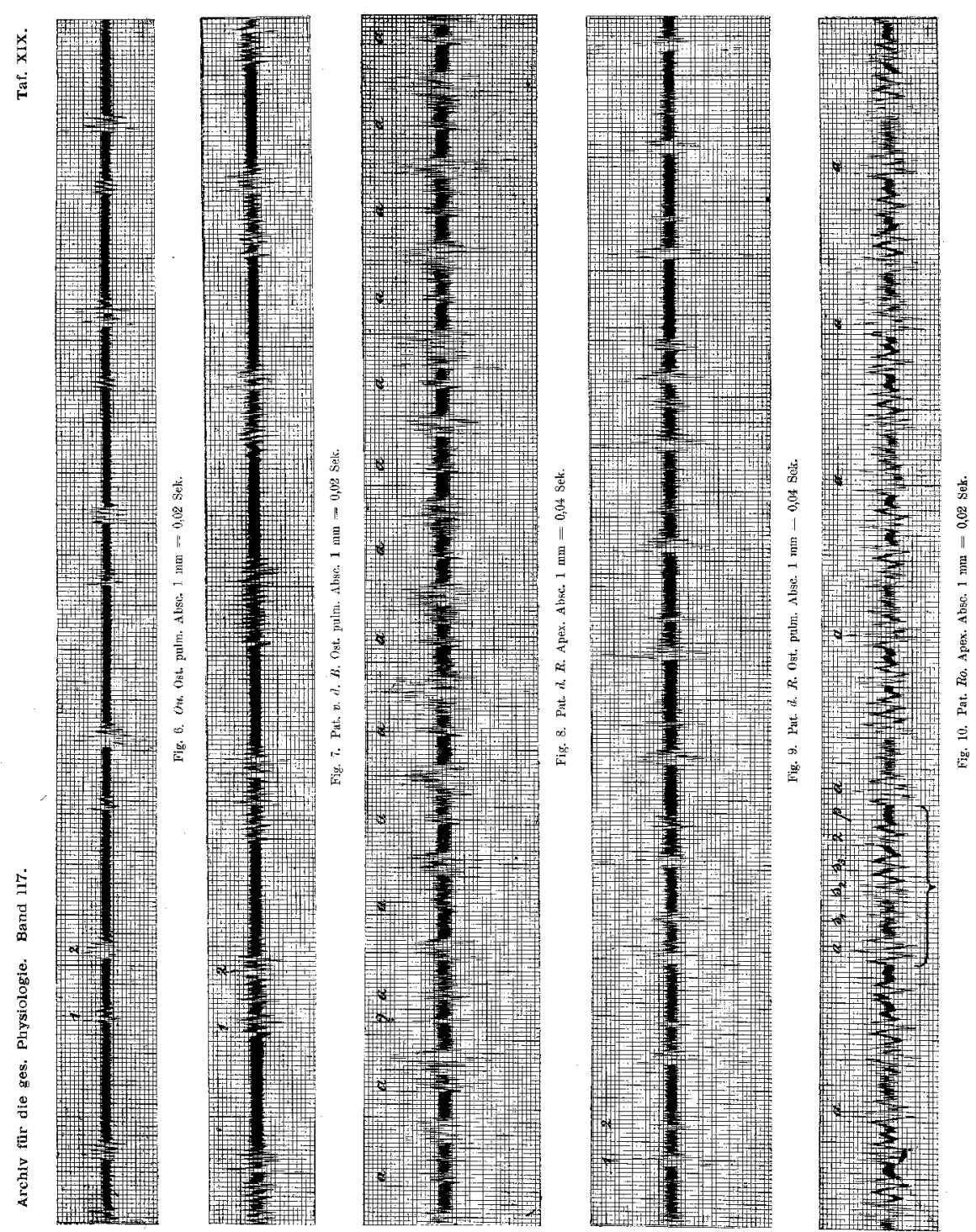